\title{
Upconversion nanoparticles with anti-Stokes luminescence as bioimaging agents
}

\author{
Polina A. Demina ${ }^{1,2,4, *}$, Evgeniy V. Khaydukovev,3, Natalia V. Sholina ${ }^{3,5}$, Vasilina V. \\ Rocheva $^{2}$, Dmitry A. Khochenkov ${ }^{5}$, Roman A. Akasov ${ }^{1,3}$ and Alla N. Generalova ${ }^{1,2}$ \\ ${ }^{1}$ Shemyakin-Ovchinnikov Institute of Bioorganic Chemistry RAS 117997, Moscow, Russia \\ ${ }^{2}$ Scientific Research Centre "Crystallography and Photonics" RAS, 119333, Moscow, Russia \\ ${ }^{3}$ I.M. Sechenov First Moscow State Medical University, 119991, Moscow, Russia \\ ${ }^{4}$ Russian Technological University (MIREA), 119454, Moscow, Russia \\ ${ }^{5}$ N. N. Blokhin National Medical Research Center of Oncology, 115478, Moscow, Russia
}

\begin{abstract}
Lanthanide-based upconversion nanoparticles attach great attention in theranostics due to their unique physicochemical and optical properties. It is innovative platform possessing peculiar properties for luminescent imaging, temperature mapping, sensing, and therapy. In present work we demonstrate advantages of new luminescent agents based on upconversion nanoparticles and hydrophylic biocompatible polymer.
\end{abstract}

\section{Introduction}

Theranoctics is a new interdisciplinary research area, which is an integration of therapy and diagnostics [1]. Upconversion nanoparticles (UCNPs) are promising nanoplatform for theranostics.[2] Lanthanide-based UCNPs are composed of inorganic crystalline ceramic host matrix, co-doped with pairs of different trivalent lanthanide ions $\left(\operatorname{Ln}^{3+}\right)$, usually the $\mathrm{Yb}^{3+}, \mathrm{Er}^{3+}$ or $\mathrm{Tm}^{3+}$. Its distinctive feature is the ability to convert near-infrared (NIR) light into higher energy visible or ultraviolet (UV) light [3]. UCNPs have been successfully used for in vivo bioimaging due to the low biotissue autofluorescence. The most common mechanism for passive nanoparticle delivery into solid tumors is the enhanced permeability and retention effect (EPR) allowing UCNPs to preferentially diffuse and accumulate in tumor tissues [4]. The efficiency of the EPR-effect in UCNP delivery into the tumor is associated with the circulation half-lifetime in blood.

In present work we developed nanocomplexes with prolonged circulation time in the blood at intravenous injection by UCNP surface modification with colominic acid (CA). $\mathrm{CA}$ is endogenous non-immunogenic, biodegradable, non-toxic compound with low serum protein adsorption [5]. These properties determine the prospects of using for solid tumour visualization in vivo. We studied chemical and photophysical properties of CA-modified UCNP agents and demonstrated its effective accumulation in tumour due to prolonged blood circulation time for 3 hours in comparison with UCNPs-polyethylene glycol complexes.

\footnotetext{
* Corresponding author: polidemina1207@yandex.ru
} 


\section{Results}

Nanoparticles are synthesized in organic solvent that determines their hydrophobic surface properties. The development of hydrophilization methods for obtaining hydrophilic UCNP probes, which can be homogeneously dispersed in an aqueous medium, biocompatible and colloidal stable in a biological environment is crucial. Firstly, we hydrophilized UCNPs through coating nanoparticles with polyethyleneimine (PEI) having NH2 groups, without removing the oleic acid from the UCNP surface. Then, we modified the surface in two different ways: 1) by forming a covalent bond between the PEI and the CA using the carbodiimide activation method (CA-CDI); 2) by adsorption of the CA on the UCNP surface due to electrostatic interactions $(\mathrm{CA}(+/-))$. The obtained samples are hydrophilic retaining their colloidal stability in $\mathrm{PBS}, \mathrm{pH}=7.2$ for 1 month. IR spectroscopy successfully confirmed the modification.

The minimal adsorption of blood proteins on the particles provided the prolonged particle circulation time in blood and effective accumulation in the tumor. Our study showed that after incubation CA-modified UCNPs with mouse serum at $\mathrm{T}=37^{\circ} \mathrm{C}$ for 1 hour the probes practically did not adsorb the serum proteins as compared to UCNPs/PEI. These data were obtained by detection the protein concentration in the supernatants using the Bradford method. Thus, CA-modified UCNPs have potential to prolonged circulation in the body.

The UCNPs accumulation in cells was studied by flow cytometry on the cell lines of murine macrophages RAW 264.7 and human monocytes THP-1. The cell suspension (106 cells per ml) was incubated with $0.1 \mathrm{mg} / \mathrm{ml}$ modified UCNPs for $30 \mathrm{~min}$ in a $\mathrm{CO}_{2}$ incubator $\left(37 \mathrm{C}, 5 \% \mathrm{CO}_{2}\right.$ ). UCNPs covered with CA-CDI showed a higher level of accumulation in the cells than $\mathrm{CA}(+/-)$.It is worth noting that the level of CA(+/-)-UCNP accumulation was comparable to the accumulation of PEG-modified UCNPs.

We studied in vivo circulation time in blood after intravenously injection in mice Balb/c and collected blood samples from the tail vein at several time intervals. The samples were analyzed using home built anti-Stokes fluorescent microscope [6]. Images from four random areas were taken and calculation of UCNP number in all areas was made.

CA coating of UCNPs made it possible to reduce the UCNP interaction with serum proteins, leading to longer circulation in the bloodstream of small animals (up to 3 hours) as compared to UCNPs, modified with PEG, which are in the bloodstream no longer than 1 hour.

The reported study was partly funded by RFBR according to the research project No. 17-03-01033.

\section{References}

1. X. Chen, S. S. Gambhir, J. Cheon Acc. Chem. Res. 44, 841 (2011)

2. G. Chen, H. Qiu, P. N. Prasad, X. Chen, Chem. Rev. 114, 5161 (2014)

3. A. N. Generalova, B. N. Chichkov, E. V. Khaydukov, Adv. Colloid Interface Sci. 245, 1 (2017)

4. J. Fang, H. Nakamura, H. Maeda, Adv. Drug Deliv. Rev. 63, 136 (2011)

5. A. I. Fernandes, G. Gregoriadis, Biochim. Biophys. Acta 1341, 26 (1997)

6. A. N. Generalova, V. V. Rocheva, A. V. Nechaev, D. A. Khochenkov, N. V. Sholina, V. A. Semchishen et al., RSC Adv. 6, 30089 (2016) 